\title{
A visual aid for cellular imaging analysis
}

A new software tool plots quantitative cell traits as cell-like glyphs.

Detecting differences in cellular morphology can be a demanding task owing to the human eye's bias or limitations in identifying subtle but important variations in cellular features. Scientists have therefore developed automated and unprejudiced ways to quantify selected cell attributes extracted from images. However, an additional challenge is presenting the analyzed data so that all parameters are easily understood and appreciated. Heat maps and graphs are the most common methods for representing imaging measurements, but none of them offers an obvious and easily interpretable representation of cellular characteristics.

Chris Bakal and his team at the Institute of Cancer Research in London now present a software tool dubbed PhenoPlot that makes quantification of complex cellular phenotypes easier to understand. PhenoPlot allows the simultaneous presentation of up to 21 quantifiable cell features by representing them as symbols (glyphs). These include features such as the widths and lengths of the cell and nucleus and the area of cell protrusions as well as variables such as the neighboring fraction, which is the portion of the cell border that is in contact with surrounding cells. One or several variables can be encoded into a glyph; for example, the cell body and nucleus are represented as ellipses, and cell protrusions as spikes. Each glyph's dimension, filling or coloring reflects the quantitative value of a variable. For example, the proportion of the cell or nuclear ellipse that is filled with a symbol represents the cellular or nuclear texture and reflects the distribution of image pixel intensity in these regions. The final presentation of the quantified cellular features bears resemblance to the cellular prototype it represents, like a cell's alter ego.
Bakal's team applied PhenoPlot to profile 19 breast cancer lines that had been fluorescently stained and imaged. They measured nine different parameters, including the size and texture of cells and nuclei, area of cell protrusions, neighboring fraction and cell 'ruffliness.' They found that cells with similar parameter values clustered in five groups and that each group's unique morphology was easily linked to cell motility and invasiveness.

PhenoPlot offers two main benefits: it eliminates bias arising from human analysis of cell images, and it offers an intuitive presentation of quantified parameters. The authors point out that although PhenoPlot is designed primarily for data extracted from cellular images, it can be used with any quantitative data.

Vesna Todorovic

RESEARCH PAPERS

Sailem, H.Z. et al. Visualizing cellular imaging data using PhenoPlot. Nat. Commun. 6, 5825 (2015). 